\title{
Estudio integral de desnutridos en un Consultorio del Area Norte
}

\author{
DRA. ANA M. KAEMPFFER *. SRA. JULIA ALARCON"*, SRTAS.: LUCY BAHAMONDES, BELIA \\ BLANCO Y OLIVLA MARTINEZ. SRES.: HERNAN FIGUEROA, SERGIO MONTENEGRO, GER- \\ MAN MUHLHAUSEN. RAUL MURANDA $\Upsilon$ MARIO NAVARRETE tht.
}

I. INTRODUCción. La mayoría de los países latinoamericanos enfrentan actualmente graves problemas de desnutrición, que inciden negativamente en las posibilidades de desarrollo de ellos.

Estos problemas se relacionan tanto con la disponibilidad global de alimentos como con su distribución, lo que hace que, aún con una disponibilidad global cercana a los requerimientos de la población, coexistan problemas de déficit (desnutrición) y de exceso (obesidad) que afectan en forma preferente a determinados niveles socioeconómicos y a determinados grupos de edad.

La población infantil es característicamente la más afectada por la desnutrición en América Latina y en Chile, en que se señala que cifras cercanas a $15 \%$ de los lactantes, y de hasta $40 \%$ de los preescolares en algunas áreas, serían desnutridos. El efecto de este estado nutritivo alterado se aprecia en las altas tasas de morbilidad y mortalidad que se observan entre los desnutridos. Así por ejemplo en estudios recientes se ha demostrado que un $50 \%$ de los niños que fallecen por diarrea aguda y un $32 \%$ de los que mueren por patología respiratoria son desnutridos. Así, si bien la desnutrición no figura en las estadísticas como causa importante de muerte, sí lo es como enfermedad sustrato, que hace que el niño desnutrido enferme más, y al enfermar lo haga con mayor gravedad y su riesgo de muerte sea muy superior al del niño bien sutrido.

Por eso se hace indispensable conocer la magnitud de la desnutrición infantil, los factores que la condicionan y los efectos que produce, para poder planificar y ejecutar los programas de control más adecuados. Un primer nivel de investigación

\footnotetext{
- Profesor Asociado Medicina Preventiva, Unidad Area Norte. Departamento de Salud Pública. Universidad de Chile.

* Asistente Socia? Docentc. Unidad Area Norte, Departamento de Salud Pjblica. Universidad de Chile.

*. Estudiantes de Medicina de III Año, Area Norte, 1972.
}

es la población infantil que se controla en Consultorios, que representa una proporción importante de los niños y en la que se reúnen condiciones de accesibilidad y de cierta cantidad de información que se recoge sistemáticamente.

Estas consideraciones llevaron a nuestro grupo a interesarse por efectuar un análisis epidemiológico de la desnutrición en un Consultorio pediátrico del Area Norte, como parte del Curso de Epidemiología de 3er. Año de Medicina.

Se eligió el Consultorio Lucas Sierra, ubicado en la comuna de Conchalí, que atiende una población de unos 90 mil habitantes, con una porporción de población infantil comparable al promedio nacional. El nivel de vida es en general bajo; la población beneficiaria del SNS es de alrededor de $70 \%$. La mortalidad infantil es inferior al promedio nacional (52\% para la comuna). Las cifras de desnutrición calculadas en el Consultorio de acuerdo a la gráfica de Mardones fluctúa entre 12 y $15 \%$ en los 4 sectores de atención.

II. Própósito. Conocer la magnitud de la desnutrición en un grupo de lactantes controlados en un Consultorio Distrital del S.N.S. y sus factores condicionantes.

\section{Objetivos:}

1. Realizar un diagnóstico adecuado de estado nutritivo en el primer año de vida en una muestra de niños controlados en Consultorio.

2. Analizar la relación entre estado nutritivo y algunos condicionantes conocidos y otros que supusimos importantes.

3. Estudiar el impacto que tiene el estado nutritivo sobre la morbilidad registrada, analizando su frecuencia, tipo de gravedad. 
4. Conocer en el terreno las condiciones socioeconómicas, culturales y familiares de un grupo de desnutridos.

III. Material y MÉtrodos. Para cumplir el primer y segundo objetivo, se tomó una muestra del $20 \%$ de niños controlados en el Consultorio del grupo lactante mayor (1-2 años).

La razón para tomar como muestra al lactantemayor fue que eso nos permitiria examinar la evolución del niño durante todo el primer año. Por lo tanto, todos los niños tienen 1 año de observa. ción. No se siguió la observación durante el $2^{\circ}$ año, ya que los controles y acciones que se desarrollan son más espaciados en el $2^{\circ}$ año de la vida, y la velocidad de crecimiento es menor.

El muestreo sistemático del fichero del Consultorio dio un total de 145 fichas. De ellas se eliminaron 6, que correspondían a niños inscritos después del año.

Las 139 fichas resultantes de nuestro muestreo fueron revisadas de acuerdo a una pauta en que se consignaron los siguientes datos:

Nombre

Ficha

Fecha nacimiento

No de orden

Edad de inscripción

Edad actual

Peso actual.

Edad
Estado civil
Escolaridad
Ocupación
Previsión

Peso y talla

Nacimiento

Talla. Peso ideal.

Padre

\% sobre el diagnóstico peso ideal

3 meses

6 meses

9 meses

12 meses.

Duración lactancia materna exclusiva (indicada por la primera indicación de suplemento lácteo).

Total controles salud 1er. año

Relación Sano/Morbilidad

Relación controles/norma

Total consultas morbilidad

Diagnósticos:

Para el cumplimiento del primer objetivo nos basamos en la clasificación de Gómez para grados de desnutrición. Esta clasificación tiene la ventaja de que se basa en el criterio de peso teorico nor- mal es decir, es en cierta forma un diagnóstico individual de estado nutritivo para cada niño que se basa en el peso de nacimiento al que se agrega un ineremento mensual observado en estudios longitudinales de niños sanos. Este sistema tiene la ventaja de eliminar del grupo de desnutridos a ninos de bajo peso de nacimiento y de no calificar de eutróficos a niños de alto peso de nacimiento, y que a pesar de incrementos ponderales insuficientes son calificados como eutróficos según criterio estadístico. Calculamos para cada niño de la muestra el peso teórico normal en los 4 trimestres del primer año.

Los objetivos 2 y 3 se cumplieron analizando las variables registradas.

Para el cumplimiento del $4{ }^{\circ}$ objetivo realizamos encuesta domiciliaria. La muestra inicial, con niños de los diversos sectores fue de 46 niños. En 15 no se pudo realizar por domicilio falso o cambio de domicilio que no había sido comunicado al Consultorio.

Las visitas se realizaron rotativamente para evitar que más de 2 personas entraran al hogar, y todas tuvieron la supervisión directa de la asistente social docente. La pauta utilizada consideró: datos de identificación; tamaño y composición del grupo familiar; otros desnutridos en la familia; condición civil de los padres, instrucción, trabajo, previsión, ingreso mensual "per cápita" para alimentación; calidad de la vivienda; alimentación del niño: quién la proporciona, corresponde a las indicaciones, técnica de preparación y administración.

IV. Resultados. A. Del análisis de fichas. 1. Diagnóstico de estado nutritivo.

Comparamos el diagnóstico que figura en las fichas del Consultorio, realizado de acuerdo a la gráfica de Mardones, con el análisis individual para cada niño, según el criterio de Gómez. En el momento de cumplir un año de edad, la situación es la que muestra la tabla $N^{\circ} 1$.

T A B L A No 1

CALIFICACION DEL ESTADO NUTRITIVO

\begin{tabular}{lrrrr}
\hline & $\begin{array}{r}\text { Según Consultorio } \\
\end{array}$ & $N^{\circ}$ & & \multicolumn{2}{c}{$\begin{array}{c}\text { Según clasifica- } \\
\text { ción de Gómez } \\
N^{o}\end{array}$} \\
\hline Eutróficos & 114 & 82,2 & 94 & 67,6 \\
Desnutridos I & 22 & 15,7 & 37 & 26,6 \\
Desnutridos II & 3 & 2,1 & 8 & 5,8 \\
Desnutridos III & - & - & - & - \\
\hline T o t a 1 & 139 & 100,0 & 139 & 100,0 \\
p $=0,01-0,02$. & & & &
\end{tabular}


Se aprecia un mayor $\%$ de niños con déficit ponderal al aplicar el criterio de Gómez que nosotros consideramos más adecuado, ya que considera el peso de nacimiento de cada niño en particular.

La diferencia aplicando el test de chi cuadrado, es significativa. El criterio de Gómez resulta evidentemente más estricto, lo que representa una ventaja para pesquisar precozmente la desnutrición e iniciar las acciones que corresponda (mayor frecuencia de controles, seguimiento domiciliario, solución de problemas socioeconómicos, etc.).

Si revisamos la calificación de estado nutritivo por trimestres los resultados aparecen en la tabla Ne 2 .

Se aprecia que el menor porcentaje de desnutrición ocurre en el primer trimestre de la vida. La cifra aumenta a partir del 40 mes. Además, llama la atención la disminución de los controles de peso en el 3er. trimestre, que además coincide con un alza en la proporción de niños desnutridos, que no se corrige en el 4 i trimestre.

Otro de los factores que se menciona a menudo en relación al riesgo infantil es la edad de
T A B L A N N $^{2}$

CALIFICACION DE ESTADO NUTRITIVO SEGUN TRIMESTRES

\begin{tabular}{|c|c|c|c|c|c|c|}
\hline \multirow{2}{*}{\multicolumn{3}{|c|}{$\begin{array}{c}\text { Trimestre Eutroficos } \\
\text { No }\end{array}$}} & \multicolumn{2}{|c|}{ Desnutridos } & \multicolumn{2}{|c|}{ Total consignado } \\
\hline & & & $N ?$ & $\%$ & $N^{O}$ & $\%$ \\
\hline 10 & 90 & 81,8 & 20 & 18,2 & 110 & 10 \\
\hline $2 \%$ & 77 & 70,6 & 32 & 29,4 & 109 & 10 \\
\hline 30 & 43 & 65,1 & 23 & 34,9 & 66 & 100 \\
\hline 49 & 84 & 56,1 & 45 & 34,9 & 129 & 100 \\
\hline
\end{tabular}

la madre. En la tabla siguiente se resume dicha información.

La menor proporción de eutróficos de madres menores de 20 y mayores de 30 años es significativa $(p=0,02-0,05)$.

Otro factor de riesgo asociado con frecuencia a la edad de Ia madre es el número de orden del nin̄o, que traduce el tamaño familiar. En la tabla siguiente se presenta la distribución de eutróficos y desnutridos frente a dicha variable.

T A B L A Nơ 3

ESTADO NUTRITIVO SEGUN EDAD DE LA MADRE

\begin{tabular}{|c|c|c|c|c|c|c|c|c|}
\hline \multirow[t]{2}{*}{ Edad } & \multicolumn{2}{|c|}{ Eutroficos } & \multirow[b]{2}{*}{ No } & \multicolumn{3}{|c|}{ Desnutridos } & \multicolumn{2}{|c|}{$T \circ t a t$} \\
\hline & $N^{o}$ & $\%$ & & $\%$ & $N P$ & $\%$ & $N^{\circ}$ & $\%$ \\
\hline o. 20 & 17 & 68,0 & 6 & 24,0 & 2 & 8,0 & 25 & 100,0 \\
\hline $20-24$ & 22 & 81,5 & 4 & 14,8 & 1 & 3,7 & 27 & 100,0 \\
\hline $25 \cdot 29$ & 12 & 85.7 & 2 & 14,3 & 一 & 一 & 14 & 100,0 \\
\hline $30-34$ & 3 & 42,9 & 3 & 42,9 & 1 & 14,2 & 7 & 100,0 \\
\hline $35 y+$ & 6 & 42,8 & 7 & 50,1 & 1 & 7,1 & 14 & 100,0 \\
\hline No consignado & 34 & 65,3 & 15 & 28,9 & 3 & 5,8 & 52 & 100,0 \\
\hline$T \circ t a l$ & 94 & 67,6 & 37 & 26,6 & 8 & 5,8 & 139 & \\
\hline
\end{tabular}

T A B L A No 4

ESTADO NUTRITIVO SEGUN NUMERO DE ORDEN DE NACIMIENTO

\begin{tabular}{|c|c|c|c|c|c|c|c|c|}
\hline \multirow[t]{2}{*}{ No de orden } & \multicolumn{2}{|c|}{ Eutroficos } & \multirow[b]{2}{*}{$N^{o}$} & \multicolumn{3}{|c|}{$D$ esnutridos } & \multicolumn{2}{|c|}{$T \circ t a t$} \\
\hline & $N^{a}$ & $\%$ & & $\%$ & $N^{Q}$ & $\%$ & $N^{o}$ & $\%$ \\
\hline 19 & 47 & 79,7 & 9 & 15,2 & 3 & 5,1 & 59 & 100,0 \\
\hline $2^{0}-4^{o}$ & 40 & 62,4 & 21 & 32,9 & 3 & 4,7 & 64 & 100,0 \\
\hline $5^{\circ}-7^{0}$ & 5 & 50,0 & 4 & 40,0 & 1 & 10,0 & 10 & 100,0 \\
\hline $89 y+$ & 1 & 25,0 & 2 & 50,0 & 1 & 25,0 & 4 & 100,0 \\
\hline No consignado & 1 & 一 & 1 & - & $\longrightarrow$ & - & 2 & 100,0 \\
\hline$T \circ t a 1$ & 94 & 67,6 & 37 & 26,6 & 8 & 5,8 & 139 & 100,0 \\
\hline
\end{tabular}


Las diferencias son significativas $(p=0,01)$.

EI análisis de las tablas Nos. 3 y 4 indica que la menor proporción de eutróficos se encuentra los hijos de madres menores de 20 y mayores de 30 años y que la desnutrición es paralela al aumento de tamaño de la familia, lo que explica el mayor porcentaje de desnutridos hijos de madres mayores de 35 años.

Los primogénitos, que en aspectos esencialmente biologicos son niños de mayos riesgo, están menos expuestos a desnutrición que los que siguen. Estos hallazgos indicarían que la condición de desnutrido en nuestra muestra se asocia más con los problemas económicos y de cuidados que implica una familia numerosa que con la inexperiencia o la ignorancia en puericultura atribuidas a la primípara, que serían suplidas por la educacion y el control realizados por el Consultorio.

En seguida nos preocupamos de analizar la influencia del estado civil y de la escolaridad de la madre en el estado nutritivo del niño.

Tenemos así que la mayor proporción de desnutridos se encuentra entre los hijos de mujeres solteras y con viveles muy bajos de escolaridad (analfabetas o menor de $4^{0}$ básico). Ambas variables son conocidas como conđicionantes de desnutrición por la frecuencia con que el hijo ilegítimo carece de la protección afectiva y económica del padre, y por la dificultad para realizar educación en salud en analfabetos.

Otro factor de importancia, por incidir directamente en el aspecto económico, es la ocupación y situación previsional del pađre. La información se entrega en las tablas siguientes.

En relación con la actividad del pađre consignada en la ficha, Ios niños desnutridos se agrupan de preferencia en la categoría "obrero no especializado", en tanto que los eutróficos tienen una

T A B L A Nu 5

ESTADO NUTRITIVO SEGUN ESTADO CIVIL DE LA MADRE

\begin{tabular}{|c|c|c|c|c|c|c|c|c|}
\hline \multirow[t]{2}{*}{ Estado civil } & \multicolumn{2}{|c|}{ Eutroficos } & \multirow[b]{2}{*}{$N^{q}$} & \multicolumn{3}{|c|}{$D e \sin { }_{D 2} i d o s$} & \multicolumn{2}{|c|}{$T \circ \operatorname{al}$} \\
\hline & $N o$ & $\mathscr{b}$ & & $\%$ & $N^{0}$ & $\%$ & No & $\%$ \\
\hline Casadas & 47 & 74,6 & 14 & 22,8 & 2 & 3,2 & 63 & 100,0 \\
\hline Solteras & 12 & 54,6 & 7 & 31,8 & 3 & 13,6 & 22 & 100,0 \\
\hline No consignado & 35 & 64,8 & 16 & 29,6 & 3 & 5,6 & 54 & 100,0 \\
\hline Tot a 1 & 94 & 67,6 & 37 & 26,6 & 8 & 5,8 & 139 & 100,0 \\
\hline
\end{tabular}

Diferencias significativas $(\mathbf{p}=0,02-0,05)$.

T A B L N No 6

ESTADO NUTRITIVO SEGUN ESCOLARIDAD DE LA MADRE

\begin{tabular}{|c|c|c|c|c|c|c|c|c|}
\hline \multirow[t]{2}{*}{ Escolaridad } & \multicolumn{2}{|c|}{ Eutroficos } & \multirow[b]{2}{*}{$N^{o}$} & \multicolumn{3}{|c|}{$D e s \| d \underset{D 2}{t r d o s}$} & \multicolumn{2}{|c|}{$T \circ t a t$} \\
\hline & $N^{o}$ & $\%$ & & $\%$ & $N^{o}$ & $\%$ & $N^{o}$ & $\%$ \\
\hline Analfabetas & 2 & 33,3 & 3 & 50,0 & 1 & 16,7 & 6 & 100,0 \\
\hline - de 49 básico & 6 & 37,5 & 9 & 56,3 & 1 & 6,2 & 16 & 100,0 \\
\hline 49 a $8^{\circ}$ básico & 31 & 75,6 & 7 & 17,1 & 3 & 7,3 & 41 & 100,0 \\
\hline Educación Media & 19 & 100,0 & - & - & - & - & 19 & 100,0 \\
\hline Otra & 2 & - & 1 & - & 一 & 一 & 3 & 100,0 \\
\hline No consignado & 34 & 62,9 & 17 & 31,5 & 3 & 5,6 & 54 & 100,0 \\
\hline$T \circ t a 1$ & 94 & 67,6 & 37 & 26,6 & 8 & 5,8 & 139 & 100,0 \\
\hline
\end{tabular}

Diferencias muy significativas. 
mayor frecuencia depadres "obrero especializado" y "empleado, profesional o técnico".

En cuanto a la previsión del padre, las diferencias no son significativas, lo que es explicable, ya que aunque los padres, no estén cubiertos por ninguna previsión, lo que sucede en alrededor de un $50 \%$ de los casos, son beneficiarios legales del
SNS para los fines đe atención médica y suplementación alimentaria de sus nin̆os.

Conociendo la importancia de la duración de la lactancia materna, investigamos el punto, fijando como término de la lactancia exclusiva la primera indicación de suplemento lácteo, por lo que los datos son aproxirnados.

TA B LA No 7

ESTADO NUTRITIVO SEGUN ACTIVIDAD DEL PADRE

\begin{tabular}{|c|c|c|c|c|c|c|c|c|}
\hline \multirow[t]{2}{*}{ Acrividad } & \multicolumn{2}{|c|}{ Eutroficos } & \multirow[b]{2}{*}{ No } & \multicolumn{3}{|c|}{ DI Desnutridos } & \multicolumn{2}{|c|}{$T \circ t a l$} \\
\hline & $N^{o}$ & $\%$ & & $\%$ & $N^{o}$ & $\%$ & No & $\mathscr{\%}$ \\
\hline Sin trabajo & 1 & - & $\ldots$ & - & 2 & - & 3 & - \\
\hline $\begin{array}{l}\text { Obrero no } \\
\text { especializado }\end{array}$ & 26 & 61,8 & 15 & 35,8 & 1 & 2,4 & 42 & 100,0 \\
\hline $\begin{array}{l}\text { Obrero especializado } \\
\text { Empleado, profesio- }\end{array}$ & 13 & 81,3 & 2 & 12,5 & 1 & 6,2 & 16 & 100,0 \\
\hline nal y técnico & 10 & 100,0 & - & - & - & - & 10 & 100,0 \\
\hline Otra & 11 & 73,4 & 4 & 26,6 & - & 7,6 & 15 & 100,0 \\
\hline No consignado & 33 & 62,2 & 16 & 30,2 & 4 & 7,6 & 53 & 100,0 \\
\hline$T \circ t a 1$ & 94 & 67,6 & 37 & 26,6 & 8 & 5,8 & 139 & 100,0 \\
\hline
\end{tabular}

Significación límite $(\mathrm{p}=0,05)$.

TA B L A No 8

ESTADO NUTRITIVO SEGUN PREVISION DEL PADRE

\begin{tabular}{|c|c|c|c|c|c|c|c|c|}
\hline \multirow[t]{2}{*}{ Previsión } & \multicolumn{2}{|c|}{ Eutróficos } & \multirow[b]{2}{*}{$N^{O}$} & \multicolumn{3}{|c|}{$D e s n u t r i d o s$} & \multicolumn{2}{|c|}{ Total } \\
\hline & $N e$ & $\%$ & & $\%$ & $N^{o}$ & $\%$ & $N o$ & $\%$ \\
\hline $\begin{array}{l}\text { Funcionario SNS } \\
\text { Servicio Seguro }\end{array}$ & 11 & 78,6 & 3 & 21,4 & - & - & 14 & 100,0 \\
\hline Social & 48 & 73,8 & 13 & 20,0 & 4 & 6,2 & 65 & 100,0 \\
\hline Sin previsión & 25 & 58,1 & 14 & 32,6 & 4 & 9,3 & 43 & 100,0 \\
\hline No consignada & 10 & 58,8 & 7 & 41,2 & - & - & 17 & 100,0 \\
\hline$T \circ t a 1$ & 94 & 67,6 & 37 & 26,6 & 8 & 5,8 & 139 & 100,0 \\
\hline
\end{tabular}

Diferencia no significativa $(\mathrm{p}=0,10-0,20)$.

T A B L A No 9

ESTADO NUTRITIVO SEGUN DURACION DE LA LACTANCIA NATURAL

\begin{tabular}{|c|c|c|c|c|c|c|c|c|}
\hline \multirow[t]{2}{*}{ Duración } & \multicolumn{2}{|c|}{ Eutróficos } & \multirow[b]{2}{*}{$N ?$} & \multicolumn{3}{|c|}{ Desnutridos } & \multicolumn{2}{|c|}{$T \circ t a l$} \\
\hline & $N o$ & $\%$ & & $\%$ & $N^{o}$ & $\%$ & $N^{?}$ & $\%$ \\
\hline - de 1 mes & 22 & 68,8 & 9 & 28,1 & 1 & 3,1 & 32 & 100,0 \\
\hline 1 a 3 meses & 37 & 65,9 & 14 & 25,1 & 5 & 9,0 & 56 & 100,0 \\
\hline+ de 3 meses & 23 & 68,7 & 8 & 25,0 & 2 & 6,3 & 32 & 100,0 \\
\hline No consignado & 13 & 68,5 & 6 & 31,5 & - & - & 19 & 100,0 \\
\hline$T \circ t a 1$ & 94 & 67,6 & 37 & 26,6 & 8 & 5,8 & 139 & 100,0 \\
\hline
\end{tabular}


Se aprecia que la corta duración de la lactancia natural es un fenómeno generalizado en nuestra muestra.

Con esta situación de lactancia corta, y el evidente riesgo de desnutrición que el hecho representa para el niño, la precocidad de la inscripción y la asistencia a controles periódicos de salud, en que se imparte la educación sobre alimentacion del niño, cobran su máxima importancia. mación.

En las tablas siguientes se sintetiza la infor-

La diferencia es significativa si se compara el grupo de niños inscritos antes de los $\mathbf{1 0}$ días con el resto $(p=0,05)$. Podría entonces considerarse que el plazo aceptable de inscripción no debe sobrepasar los 10 días.

En primer lugar, se puede apreciat que, en general, el cumplimiento del programa de nin̆o sano es deficiente, ya que según normas programáticas del SNS se debería aceptar como aceptable una cifra no inferior a 10 controles al año.

La tabla muestra que la proporción de eutróficos aumenta hasta el grupo con 6 a 9 controles.
En cambjo, los desnutridos aumentan en el grupo con más de 12 controles lo que sugiere que el programa de mayor frecuencia de controles al desnutrido que tiene el Consultorio se está cumpliendo.

Investigamos a continuación la frecuencia de consultas por morbilidad y la gravedad de los cuatro clínicos diagnosticados. Los resultados se presentan en las tablas siguientes:

Se aprecia que en el grupo de desnutridos un $48,6 \%$ consulta por patología 60 más veces en el año. Las normas programáticas del SNS indican como cifra esperada de consultas de morbilidad en el lactante 6 al año. Es decir, casi la mitad de los desnutridos consultan más por patología de lo que podría calificarse como un promedio aceptable. Para nuestra muestra el promedio de consultas por patología fue de 4,5 para los eutróficos; 5,5 para los desnutridos grado I y 6,4 para los desnutridos grado II. Las diferencias observadas tienen una significación límite $(\mathrm{p}=0,05-0,010)$.

Además de la frecuencia de consultas por patología nos interesó calificar la gravedad de los cuadros mórbidos.

TABL A No 10

ESTADO NUTRITIVO SEGUN EDAD DE INSCRIPCION EN CONSULTORIO

\begin{tabular}{|c|c|c|c|c|c|c|c|c|}
\hline \multirow[t]{2}{*}{ Edad de inscripción } & \multicolumn{2}{|c|}{ Eutróficos } & \multirow[b]{2}{*}{$N^{O}$} & \multicolumn{3}{|c|}{ Desnutridos } & \multicolumn{2}{|c|}{$T \circ t a l$} \\
\hline & $N^{p}$ & $\%$ & & ${ }^{D 1} \%$ & $N^{o}$ & $\%$ & $N^{o}$ & $\%$ \\
\hline - de 10 días & 34 & 79,0 & 7 & 16,3 & 2 & 4,7 & 43 & 100,0 \\
\hline $10-14$ dias & 14 & 60,9 & 8 & 34,8 & 1 & 4,3 & 23 & 100,0 \\
\hline $15-29$ días & 15 & 57,7 & 9 & 34.6 & 2 & 7.7 & 26 & 100,0 \\
\hline 30 días $\mathrm{y}+$ & 29 & 65,9 & 12 & 27,3 & 3 & 6,8 & 44 & 100,0 \\
\hline No consignado & 2 & - & 1 & - & 一 & - & 3 & 100,0 \\
\hline$T \circ t a 1$ & 94 & 67,6 & 37 & 26,6 & 8 & 5,8 & 139 & 100,0 \\
\hline
\end{tabular}

TA B L A Nọ 11

ESTADO NUTRITIVO SEGUN NUMERO DE CONTROLES DE SALUD EN EL PRIMER ANO DE VIDA

\begin{tabular}{|c|c|c|c|c|c|c|c|c|}
\hline \multirow[t]{2}{*}{$N^{o}$ de controles } & \multicolumn{2}{|c|}{ Eutróficos } & \multirow[b]{2}{*}{$N^{o}$} & \multicolumn{3}{|c|}{ Desnut $\underset{D 2}{i} d$ os } & \multicolumn{2}{|c|}{ Total } \\
\hline & $N^{o}$ & $\%$ & & $\%$ & $N^{\circ}$ & $\%$ & $N^{o}$ & $\%$ \\
\hline - de 3 & 10 & 50,0 & 8 & 40,0 & 2 & 10,0 & 20 & 100,0 \\
\hline 3 a 5 & 24 & 60,0 & 12 & 30,0 & 4 & 10,0 & 40 & 100,0 \\
\hline 6 a 9 & 48 & 81,4 & 9 & 15,2 & 2 & 3,4 & 59 & 100,0 \\
\hline $10-12$ & 9 & 64,4 & 5 & 35,6 & - & - & 14 & 100,0 \\
\hline Más de 12 & 3 & 50,0 & 3 & 50,0 & 一 & - & 6 & 100,0 \\
\hline Tot a 1 & 94 & 67,6 & 37 & 26,6 & 8 & 5,8 & 139 & 100,0 \\
\hline Promedio & & & & 5,5 & & & & \\
\hline
\end{tabular}




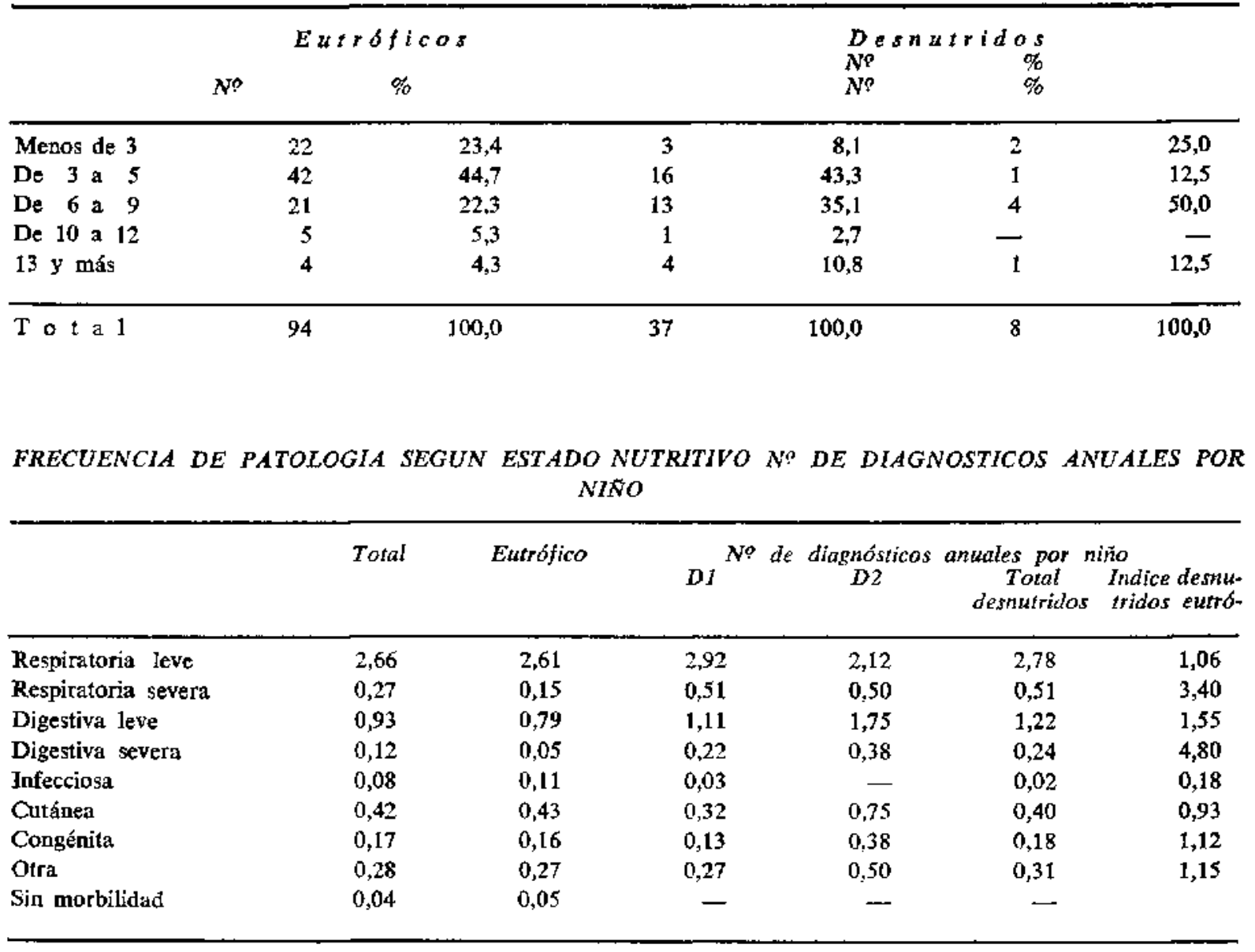

Consideramos como severos en el caso de patología respiratoria las neumopatías agudas, bronconeumonia, bronquiolitis, Jaringitis, otitis media supurada febril y para el caso de digestivas la diarrea aguda con deshidratación y la enterocolitis diagnosticadas como posibles shigelosis.

La investigación nos demostró que el grupo de desnutridos no sólo enferma con mayor frecuencia que el de eutróficos, sino que la proporción de cuadros severos es muy superior.

A continuación sintetizamos algunos de los factores condicionantes estudiados, y su significación.

\section{B. De la encuesta domiciliaria}

La segunda parte de este trabajo se realizó en terreno. Se visitó y encuestó a un grupo de 31 desnutridos de diferentes sectores del Consultorio. Para tal efecto se utilizó la encuesta descrita previamente, que fue preparada y discutida por el grupo completo, lo que facilitó su aplicación. La acogida de las familias entrevistadas fue favorable, lo que a nuestro juicio indica que tienen una buena relación con el personal de terreno del Consultorio.

Los resultados de esta investigación se exponen a continuación:

a) Estado civil de la pareja. Encontramos que 7 de las 31 madres entrevistadas eran solteras. Esta cifra (23\%) es superior al promedio nacional; en nuestro análisis previo se encontraron diferencias significativas en el riesgo de desnutrición según estado civil. Se confirma así el hecho conocido de que la falta de protección económica, social y afectiva que representa la familia incide en mayores riesgos para el bienestar del niño.

b) Nivel educacional de los padres. Es casi innecesario destacar la trascendental importancia que tiene la educación de los padres en relación al cuidado del niño. A mayor instruoción corres- 


\begin{tabular}{|c|c|c|c|c|c|}
\hline$-\quad \ldots \ldots-\cdots$ & & $\begin{array}{l}\% \text { de des- } \\
\text { nutricionn }\end{array}$ & Indice & & Variación \\
\hline Total & & 32,2 & 100 & & \\
\hline \multirow[t]{3}{*}{ Edad de la madre } & -20 años & 32,0 & & 99 & Significativa \\
\hline & $20-29$ años & 17,1 & & 53 & $p: 0,02-0,05$ \\
\hline & $30 y+$ & 57,1 & 176 & & \\
\hline \multirow[t]{4}{*}{ Orden de nacimiento } & ler. bijo & 20,3 & & 63 & Significativa \\
\hline & $2^{\circ}-4^{\circ}$ & 37,6 & 116 & & \\
\hline & $5 \%-79$ & 50,0 & 154 & & $p: 0,01$ \\
\hline & $89 y+$ & 75 & $23 i$ & & \\
\hline \multirow[t]{2}{*}{ Estado civil de la madre } & Casada & 25,4 & & 78 & Significativa \\
\hline & Soltera & 45,4 & 140 & & $\mathrm{p}: 0,02-0,05$ \\
\hline \multirow[t]{3}{*}{ Escolaridad de la madre } & Menos de $4^{0}$ básico & 63,7 & 197 & & Significativa \\
\hline & 49 - $8^{\circ}$ básico & 24,4 & & 75 & p: $-0,001$ \\
\hline & Educación Media & 0,0 & & 0 & \\
\hline \multirow[t]{4}{*}{ Actividad del padre } & Obrero no especializado & 38,2 & 118 & & Significación limite \\
\hline & Obrero especializado & 18.7 & & 58 & $p: 0,05$ \\
\hline & Empleado, profesional, técrico & 0 & & 0 & \\
\hline & Otra & 26,6 & & 82 & \\
\hline \multirow[t]{2}{*}{ Edad de inscripción en cons. } & -10 dias & 21,0 & & 67 & Signif. límite \\
\hline & $10 \mathrm{y}+$ días & 38,7 & 119 & & p: 0,05 \\
\hline \multirow[t]{2}{*}{ Nọ de controles sanos 1.er año } & -6 & 43,2 & 113 & & Significativa \\
\hline & $6 y+$ & 24,0 & & 74 & p: $0,02-0,05$ \\
\hline
\end{tabular}

ponde, lógicamente una mayor solvencia de los padres para guiar el buen desarrollo físico e intelectual del niño.

El porcentaje más alto del grupo de padres se encuentra en el nivel de $4^{\circ}-8^{\circ}$ básico; a continuación el grupo con 1 o más años de enseñanza media. En cuanto a las madres, se observa una situación menos favorable, en el sentido de que una buena proporción son analfabetas o tienen menos de $4^{\text {ọ }}$ básico, instrucción que no es suficiente según pudimos apreciar en esas madres para leer y comprender las indicaciones dietéticas escritas con que se refuerza la educación oral, ni las indicaciones de dosis y fraccionamiento de drogas (1/4 cada 6 horas por ejemplo) por desconocer medidas de peso y volumen y fracciones, que son materias que no se enseñan en los primeros años de educación básica, además de un vocabulario reducido y fuectemente influído por factores culturales no modificados por la instrucción formal.

c) Trabajo del padre. Encontramos un 20\% de padres cesantes, en contraste con el grupo de niños eutróficos en que no los hubo.

Como es obvio, esta condición trae aparejada, la inseguridad, falta de ingresos, inestabilidad e insatisfacciones que no facilitan de ninguna manera la protección física del menor.

Un resultado inesperado fue el encontrar un mayor número de obreros especializados frente a los no especializados dentro del grupo de lactantes desnutridos, en el grupo "obreros" que tenían trabajo en el momento de la encuesta.

d) Prevision de los padres. Se observó que el rubro sin previsión es el más elevado en los padres $(41,1 \%)$, lo que presupone disminución de ingresos económicos, trabajos inestables o esporádicos, e inseguridad social, (subsidios, asignaciones familiares, gratificaciones, aguinaldos, etc.).

En lo que respecta al grupo de madres, la falta de previsión era esperada, porque en su mayoría ejecutan trabajos del hogar, no remunerados. En todo caso, esta situación redundaría en beneficio, por el cuidado y atención, del niño, pero desde el punto de vista económico no lo es, como podremos apreciarlo en la tabla siguiente:

TA B L A No 15

INGRESO PER CAPITA MENSUAL PARA ALIMENTACION (1972)

\begin{tabular}{lcc}
\hline & $N^{q}$ & $\%$ \\
\hline Menos de Eo S00 & 18 & 58 \\
De E 500 a E 1.000 & 9 & 29 \\
De E 1.000 a E 2.000 & 2 & 6,2 \\
Más de E 2.000 & 0 & - \\
No consignado & 2 & 6,2 \\
\hline
\end{tabular}


Este recuento fue realizado especificamente y mediante diversas preguntas y contra preguntas bacia la disponibjilidad económicá para alimentación del grupo familiar, permitiendo la correlación con el estado nutritivo del niño.

L.as cifras que dan los resultados expuestos, son de una elocuencia indiscutible: de las $31 \mathrm{fa}-$ milias estudiadas, $18(58 \%)$, disponian para alimentación ingresos inferiores a $\mathrm{E}^{0} \mathbf{5 0 0}$ per-cápita mensual. La ración modelo del SNS, es decir la alimentación mínima indispensable para una dieta suficiente y balanceada tenía un costo aproximado de Eọ 900 en 1972. Si bien el costo de la racion modelo es un promedio bastante influido por la alimentación de los adultos, que es más cara, no lo es menor que casi un $60 \%$ de las familias no alcanzan a la mitad de esa cifta, lo que repercute en la alimentación del niño tan pronto deja de alimentarse exclusivamente de leche ( $4^{\circ}$ ó $6{ }^{\circ}$ mes). Además, por razones culturales, la distribución intrafamiliar de los alimentos disponibles es anómala y favorece notoriamente al adulto, y especialmente al jefe de familia.

e) Calidad de la vivienda. Las condiciones materiales de la vivienda que rodean al menor, en el rango "no adecuada", alcanza a $67,7 \%$. Sólo un $32,3 \%$ tenían una casa unifamiliar sólida. Sin embargo, en varias, de estas últimas las condiciones higiênicas eran extremadamente deficientes, por lo que podríamos concluir que en general hay una importante asociación entre mala vivienda $y$ desnutrición.

El $42 \%$ dispont́an de una pieza dormitorio para un grupo familiar de 4 o más personas y un $48,4 \%$ contaban con dos piezas dormitorio. Un $61,4 \%$ de las familias contaban con 1 cama para 2 o más personas.

En suma, el grado de hacinamiento y promiscuidad son importantes lo que involucra una serie de riesgos y aumento de enfermedades infecciosas, parasitarias, etc., relacionadas con esta condición de vida.

Al examinar las condiciones de saneamiento básico, encontramos que 7 de las 31 viviendas $(22,6 \%)$ no tenían agua potable y $20(64,5 \%)$ tenían pozos negros en muy malas condiciones. sin tapa, malolientes y rodeados de moscas con el evidente riesgo que ello sígnifica no sólo para los niños sino para todo el grupo familiar.

Otro rubro que se investigó fue el de la alimentación tanto en los aspectos de los aportes de nutrientes como en la técnica de preparación y administración.

Se encontró que sólo el $45 \%$ de la alimentación láctea era adecuada.

Con respecto a la alimentación no adecuada, (55\%) se destacó que el error más común se encontraba en el volumen, debiéndose posiblemente, a una mala comprensión de las indicaciones reci- bidas en el Consultorio. A este defecto sucede la irregularidad en el horario, pudiendo atribuirse a ausencias de la madre en las horas de alimentación del niño, falta de reloj $u$ otro medio de guía, o por asignársele poca importancia a la regularidad de las comidas.

La falla de la frecuencia es inferior.

Respecto de la alimentación no láctea, el déficit incide en la calidad y no en el número de comidas en el dia. La alimentación no láctea con frecuencia consiste en sopas muy líquidas, de escaso valor calórico y proteico, que no cumplen con las condiciones de consistencia y valor calórico y proteico que figuran en las pautas en uso en el Area Norte. Ello se liga no sólo a la insuficiencia de recursos económicos, tan frecuente en el grupo de desnutridos, sino a la menor asistencia a controles, donde se imparte la educación en alimentación, y a opiniones culturalmente condicionadas sobre la importancia y el valor del caldo $y$ la sopa en la alimentación infantil.

En cuanto a técnica de preparación se tomó en cuenta el tipo de mamadera usada, el aseo de los utensilios, protección contra las moscas, etc., de acuerdo a las indicaciones que se imparten en el Consultorio. En la mitad de los casos encontramos fallas de importancia en este aspecto.

Con respecto a la administración de la alimentación, en 5 niños la alimentación la preparaba y administraba la abuela o uno de los otros niños de la familia.

\section{RESUMEN}

Se presentan los resultados de la investigación realizada en una muestra de niños controlados en el Consultorio Lucas Sierra del Area Norte.

Conviene recalcar en primer lugar que la muestra adolece de una manificsta selección. Los niños estudiados han sobrevivido hasta el año de edad y se encuentran controlados en el Consultorio. Se han excluido los fallecidos en el primer año de vida, que, obviamente, no figuran en el fichero activo del Consultorio, y los no controlados. Además, en nuestra muestra no figuran desnutridos de $I I I$ grado, que por la politica asistencial del área se hospitalizan o se controlan en un Consultorio especializado.

Es por eso que al enunciar nuestros objetivos circunscribimos el estudio $y$, por lo tanto, las conclisiones a que llegamos, a niños controlados en Consultorio del SNS.

Los elementos de selección en este caso opepan en sentido favorable para los niños que forman la muestra.

1. Las cifras de desnutrición considerando el déficit en relación al peso teórico normal (criterio 
de Gómez) son mucho más altas que si se williza la gráfica de Mardones, en uso en los Consultorios del SNS.

2. El menor porcentaje de desnutridos se observa en el primer trimestre, la cifra comienza a aumentar rápidamente a partir del $4^{\circ}$ mes.

3. La mayor cifra de eutróticos se observa entre los hilos de madres entre 20 y 30 años.

4. La proporción de desnulridos es mayor entre los niños de familias numerosas $(5 y+$ hijos).

5. El estado civil y la escolaridad de la madre tienen wha gran influencia en el estado nutritivo del niño.

6. La corta duración de la lactancia natural se presenta tanto en eutróficos como en desnutridos.

7. La inscripción precoz del niño (menos de 10 dias de edad) se asocia con menor riesgo de desnutrición.

8. El promedio de controles de salud es superior en niños eutróficos o con un grado leve de deesnutrición.

9. Los desnurridos enferman con mayor frecuencia y gravedad que los eutróficos.

10. En la investigación domiciliaria comprobamos la importancia del bajo ingreso, la baja escolaridad y las malas condiciones de vivienda y saneamiento en el grupo de desnutridos.

\section{BIBLJOGRAFÍA}

1.-Dr. C. Duffau y cols. Investigaciones en lactancia cia Materna. Pediatría, Vol. 10: 102, 1967.

2.-Dres. O. Uandurraga, A Patri, J. Torres, J. Cosin, $R$. Ramirez. Estudio antropométrico de la población infantil perteneciente al Area Norte de Santiago. Pediatría Vol. 11: 278, 1968.

3.-Dr. A. Riquelme. La alimentación en Latinoamérica. Cuadernos Médico-Sociales. Marzo 1966: 5.

4.-Dres. J. Margozzini, S. Gonzúlez, O. Uniurraga. Desnutrición et un consultorio distrital. Evaluación de las acciones de prevención. Pediatría. Vol. 11; $279,1968$.
5.-Dres. J. Torres, J. Cosin, S. Gonzalez. Riesgo de desnutrición infantil. Pediatría Vol. 12: 129, 1968.

6.-Dr. F. Monckeberg. Efectos sobre el desarrollo psicomotor đel niño. CuadernosMédico-Sociales. Diciembre 1968; 5 .

7.-Richard H. Barres. Desnutrición precoz y desarro1lo mentál una relación seriamente mal entendida. Cuadernos Médico-Sociales. Marzo 1970: 23.

8.-Dres. E. Medina, y A. Kaempffer. Nutrición. Capítulo 8: "Elementos de Salud Pública". Publicaciones Unidad Area Norte. Departamento de Salud Pública y Medicina Social. Facultad de Medicina, Universidad de Chile, 1972.

9.-- Nelson, Vaughan y Mc Kay. Trastornos de la Nutrición. Tratado de Pediatría. Tomo 1 Salyat, Editores, 1971.

10.- Nutricion. Capítulo VI de "Pediatría" del Prof. J. Meneghello. Editorial Intermédica, 1972.

\section{SUMMARY}

Results of field study designed for teaching purposes are reported.

The study was penformed in order to know the undernutrition rates among infants seen at a periferic clinic in North Arca, Santiago, Chile.

It is pointed out that the sample is obvjous biased and relates only to children that have been under regular control and who have survived at least one year. Both conditions operate in favor of our sample us compared with total infant population.

Undernutrition was found to be associated with age with the lowest rate at the first trimester and a rapid increase from the fourth month on; with the member of children in the family; maternal marital status and education; low income and poor housing.

A short time of breast feeding was observed both in well nourished and under nourished babies.

Early registration (under 10 days) and frequent controls were found to be important to ge a well nourished infant.

As expected. mal nourished children have a higher and for more severe morbidity than normal ones.

We think field studies of this type are a very good teaching tearning experience to integrate clinical and public health aspects of a health problem. 